\title{
Fractal-Based Thinned Planar-Array Design Utilizing Iterative FFT Technique
}

\author{
Wentao Li, ${ }^{1}$ Yongqiang Hei, ${ }^{2}$ Jing Yang, ${ }^{1}$ and Xiaowei Shi ${ }^{1}$ \\ ${ }^{1}$ School of Electronic Engineering, Xidian University, Shaanxi, Xi'an 710071, China \\ ${ }^{2}$ State Key Laboratory of Integrated Services Networks, Xidian University, Shaanxi, Xi'an 710071, China
}

Correspondence should be addressed to Wentao Li, wtli@mail.xidian.edu.cn

Received 18 March 2012; Revised 10 July 2012; Accepted 24 July 2012

Academic Editor: Stefano Selleri

Copyright (C) 2012 Wentao Li et al. This is an open access article distributed under the Creative Commons Attribution License, which permits unrestricted use, distribution, and reproduction in any medium, provided the original work is properly cited.

\begin{abstract}
This paper investigated the synthesis problem of fractal-based thinned planar arrays by using the iterative Fourier technique (IFT). Since an inverse discrete Fourier transform relationship exists between the array factor and the element excitations for a periodic array antenna, an iterative Fourier technique is introduced to derive the array element excitation from the prescribed array factor based on this peculiar property. However, considering the IFT technique is sensitive to the initial values, the fractal-thinned array technique is employed and combined in the synthesis process to exploit the advantages of these two techniques. Moreover, the DRR of the excitation amplitude is controlled to achieve a reduction of the cost of the feeding network. Numerical examples have been carried out to validate the proposed approach.
\end{abstract}

\section{Introduction}

Thinned array has the advantages of reducing the element count, cost, overall weight, power consumption, and complexity for the filled antenna arrays. These attributes make it an attractive choice for array designs. Unfortunately, this technique may suffer from the disadvantage of high sidelobe level. Over the years, there has been a considerable amount of interest in looking into nature for inspiration when seeking new ways to solve such complex design problems. One of them is known as the fractal geometry, which was originally inspired by studying the shapes of natural objects such as trees, leaves, terrain, coastlines, cloud boundaries, and snowflakes $[1,2]$. With the use of fractals in electromagnetics, those self-similar geometries were employed in designing fractal elements [3-7] as well as arrays [8-14]. Kim and Jaggard first introduced the fractal concepts in antenna array design [8]. In [9], by using the fractal concepts, the design of multiband Koch linear arrays as well as low sidelobe Cantor linear arrays are presented. The Peano-Gosper (PG) space-filling curve has recently been exploited in the design of triangular lattice arrays with additional desirable characteristics [10] and in [11], by introducing perturbations into basic recursive array-generation scheme, the beam-steering capabilities of the Peano-Gosper array has been further enhanced. Planar array configurations based on Sierpinski carpets have been considered in [15-17]. Essentially, the fractal-based arrays are quasi-random arrays, which can be considered as the combination of the random sparse arrays and the periodic arrays. Therefore, the advantages of the random sparse arrays and the periodic arrays have been inherited in the fractal-based thinned arrays, that is, periodic arrays posses the main advantage of relatively low sidelobes, while the superiority of random arrays is robust. In other words, compared with other types of thinned arrays, the fractal-based thinned array is more robust and easier to implement and calibrate in practice.

Recently, the synthesis of antenna arrays have been carried out by many evolutionary optimization algorithms for convenience and flexibility, such as genetic algorithm, particle-swarm optimization algorithm, and differential evolutionary algorithm [18-24]. However, it is known to us all that these optimization algorithms suffer from heavy computational cost.

Since an inverse discrete Fourier transform relationship exists between the array factor and the element excitations for a periodic array antenna, Keizer proposed a new iterative Fourier technique, which was used iteratively to derive the 
array element excitations from the prescribed array factor based on this peculiar property. Furthermore, this method has been successfully applied in the synthesis of linear arrays [25] and planar arrays [26]. The unique characteristic of this algorithm is the low computation time that is not related to the size of the array when obtaining the prescribed results, but mainly depends on the level of the prescribed requirements. Thus, this algorithm is especially suited for large arrays.

This paper focuses on synthesizing large fractal-based thinned arrays to achieve low sidelobe level by exploiting the advantages of both the fractal arrays and the IFT method. By controlling the weights of the array elements, we attempt to increase the degrees of freedom in the design process, and achieve significant improvements to the array parameters by controlling the weights of the array elements. Besides, the Sierpinski carpet-patterned array is employed and lower sidelobe level is obtained by using the IFT method. Moreover, the dynamic range ratio (DRR) of the excitation amplitudes is also controlled for easy realization in practical engineering. Numerical examples show the effectiveness and superiority of the IFT-based technique.

The remainder of the paper is organized as follows. Section 2 briefly explains the formulation of the problem. Section 3 presents the detailed architecture of the proposed algorithm. Numerical results are discussed in Section 4, while Section 5 concludes this paper.

\section{Problem Formulation}

2.1. Conventional Planar Array Antenna. When aperture edge effects are neglected, that is, all array elements are in the same array environment, the far field of a planar array consisting of $M \times N$ elements arranged along a rectangular lattice with an element spacing distance $d_{x}$ in the $x$ direction and $d_{y}$ in the $y$ direction as shown in Figure 1, can be written as follows:

$$
\begin{gathered}
F(u, v)=\operatorname{EF}(u, v) \operatorname{AF}(u, v) \\
\mathrm{AF}(u, v)=\sum_{m=0}^{M-1} \sum_{n=0}^{N-1} A_{m n} e^{j k\left(m d_{x} u+n d_{y} v\right)}
\end{gathered}
$$

where EF and AF are the embedded element pattern and array factor, respectively. $A_{m n}$ is the complex excitation, $k=$ $2 \pi \lambda^{-1}$ with $\lambda$ the wavelength

$$
\begin{aligned}
& u=\sin \theta \cos \varphi, \\
& v=\sin \theta \sin \varphi .
\end{aligned}
$$

Visible space is defined as $u^{2}+v^{2} \leq 1$.

The DRR of the excitation amplitudes is defined as

$$
\mathrm{DRR}=\frac{\max _{m, n}\left\{\left|A_{m, n}\right|\right\}}{\min _{m, n}\left\{\left|A_{m, n}\right|\right\}}
$$

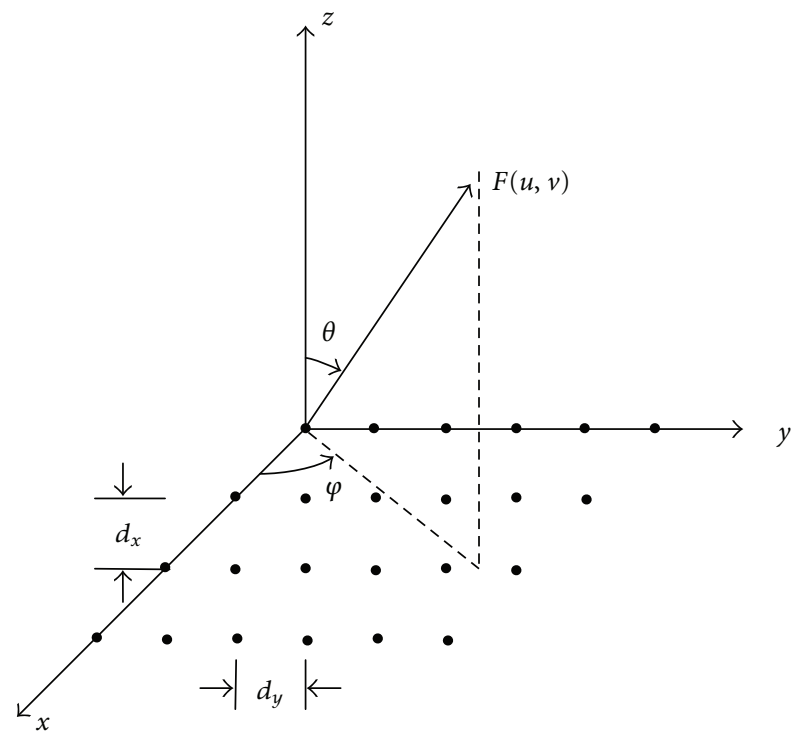

Figure 1: Geometry of $M \times N$-element planar array on the $x-y$ plane.

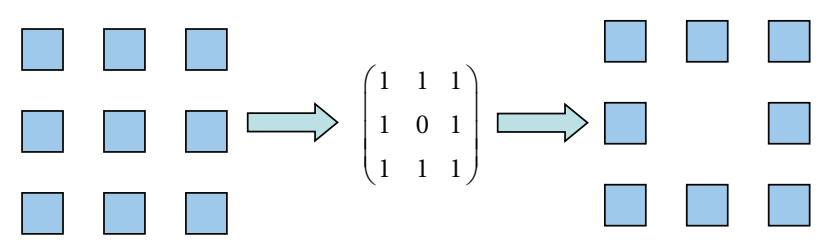

FIGURE 2: Illustration of the procedure for converting a $3 \times 3$ planar array to the first level $(P=1)$ of Sierpinski carpet based-array.

2.2. Fractal Planar Array Antenna. Fractal arrays can be formed recursively through the repetitive application of a generating array, in which larger arrays at higher levels (i.e., $P>1$ ) can be recursively constructed by a small array at level one $(P=1)$. In many cases, the generating subarray has elements that are turned on and off in a certain pattern. A set formula for copying, scaling, and translating of the generating array is then followed in order to produce a family of higher order arrays. Thus, fractal arrays that are created in this manner consist of a set of self-similar subarrays.

In this paper, the basic Sierpinski carpet array, as shown in Figure 2, is investigated. It can be created by starting with three-element-generating subarray, and then applying it repeatedly over $P$ scales of growth [14].

The generating subarray in this case has uniformly spaced elements, with the center element turned off or removed. Various stages of growth of the Sierpinski carpet array are shown in Figure 3. For the second-stage antenna array the number of elements due to Sierpinski-carpet-based arraythinning procedure is 64 instead of 81 for the planar Euclidian array. This provides for a $21 \%$ reduction in number of elements. 


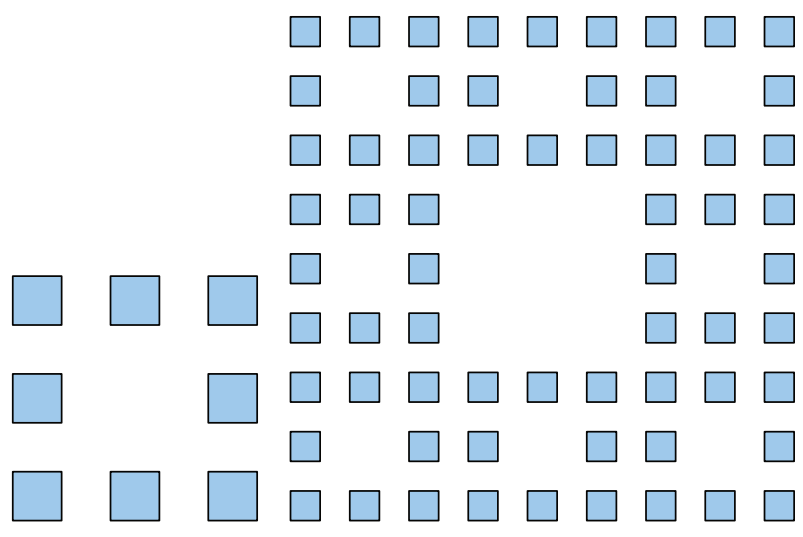

(a)

(b)

FIGURE 3: Graphical illustration of the first two stages in the construction of the Sierpinski carpet array, (a) stage 1 and (b) stage 2.

\section{Iterative Fourier Technique}

From Section 2.1, we know that the right-hand part of (2) forms a truncated double Fourier series, which relates the element excitation coefficients of the array to its array factor through a discrete inverse Fourier transform. Therefore, a direct FFT applied on AF will yield the excitation coefficient. From the property of the Fourier transform, we know that the array factor AF is periodic in $u$ dimension over the interval $\lambda / d_{x}$ and periodic in $v$ dimension over the interval $\lambda / d_{y}$.

The array factor can be calculated with a $K \times K$ point inverse FFT, with $K$ equal to an even power of 2 and using zero padding when $K>\max (M, N)$, the $K \times K$ positions of the AF samples in $u-v$ space will be

$$
\begin{aligned}
& u_{p}=\frac{p}{K} \frac{\lambda}{d_{x}}, \quad p=-\frac{K}{2} \cdots \frac{K}{2}-1, \\
& v_{q}=\frac{p}{K} \frac{\lambda}{d_{y}}, \quad q=-\frac{K}{2} \cdots \frac{K}{2}-1 .
\end{aligned}
$$

Implementation of the IFT algorithm for fractal based planar arrays proceeds as follows with the flowchart shown in Figure 4.

Step 1. Start the synthesis using a random initialization for element excitations and the basic array structure is as the Sierpinski carpet array.

Step 2. Compute AF from $\left\{A_{m n}\right\}$ using a $K \times K$ point $2 \mathrm{D}$ inverse fast Fourier transform (IFFT) with $K>\max (M, N)$ by zero padding the excitation to obtain the required number of points.

Step 3. Adapt AF to the prescribed maximum constraints and compute $\left\{A_{m n}\right\}$ for the adapted AF using a $K \times K$ point 2D forward FFT.

Step 4. Truncate $\left\{A_{m n}\right\}$ from $K \times K$ samples to $M \times N$ samples by making all samples outside the array zero.

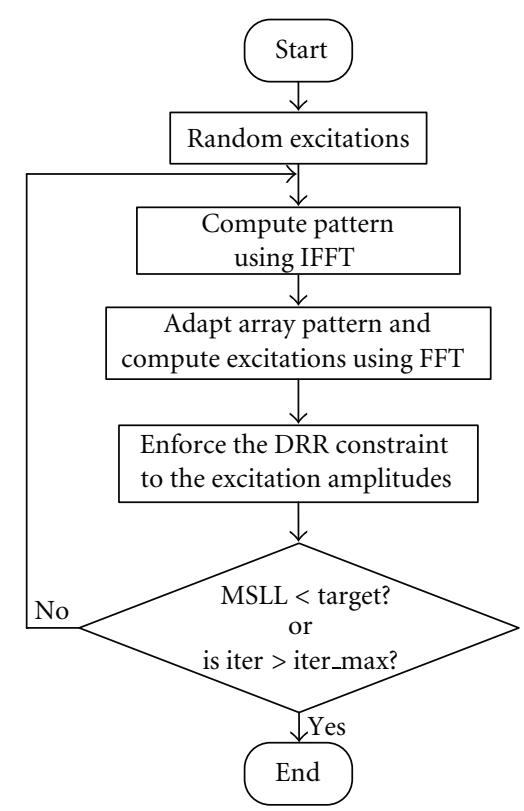

FIgure 4: Flowchart of the iterative Fourier technique for fractalbased planar-array pattern synthesis.

Step 5. Set the magnitude of the excitations lower than the preset value to zero to obtain the modified fractal-thinned array. Then set the other magnitude of the excitations violating the dynamic-range constraint to the lowest permissible value.

Step 6. Repeat Steps 2-5 until the sidelobe requirements are satisfied, or the maximum number of iterations is reached.

\section{Numerical Results}

In this part, two examples will be simulated to show the superiority and effectiveness of the presented approach, which consists of uniformly spaced isotropic elements and the interelement distances are $d_{x}=d_{y}=0.5 \lambda$. For both of these two examples, the preset value in Step 5 is $20 \mathrm{~dB}$.

Now let us consider the traditional calculation approach of the array pattern by using the element-by-element superposition principle, in which the complexity is determined by the total number of array elements and the far-field directions to be considered. Therefore, it is very time-consuming. While in the IFT technique, array pattern is computed through an FFT operation. Especially, for the FFT method, the computational time is only determined by the sampling points and the computational complexity can be reduced significantly. This property is very important for large array synthesis. Table 1 compares the average times required for 100 independent evaluations of planar array patterns when using these two methods. It is observed that the simulation time can be reduced dramatically by using the 2D FFT method.

The first example is the stage $3(P=3)$ Sierpinski carpet array. The IFT method to obtain the element distributions operates with $1024 \times 1024$ point forward and inverse FFTs. 
TABLE 1: Average-time comparison for computing the planar-array pattern with different methods.

\begin{tabular}{lcccccc}
\hline \multirow{2}{*}{ Number of elements } & \multicolumn{3}{c}{ Element-by-element superposition(s) } & \multicolumn{3}{c}{2 DFT(s) } \\
& $\begin{array}{c}512 \times 512 \\
\text { points }\end{array}$ & $\begin{array}{c}1024 \times 1024 \\
\text { points }\end{array}$ & $\begin{array}{c}2048 \times 2048 \\
\text { points }\end{array}$ & $\begin{array}{c}512 \times 512 \\
\text { points }\end{array}$ & $\begin{array}{c}1024 \times 1024 \\
\text { points }\end{array}$ & $\begin{array}{c}2048 \times 2048 \\
\text { points }\end{array}$ \\
\hline $9 \times 9$ & 13.3388 & 48.4308 & 194.4324 & 0.0169 & 0.0781 & 0.3501 \\
$27 \times 27$ & 182.7668 & 694.7960 & 2862.5529 & 0.0141 & 0.0831 & 0.3611 \\
\hline
\end{tabular}

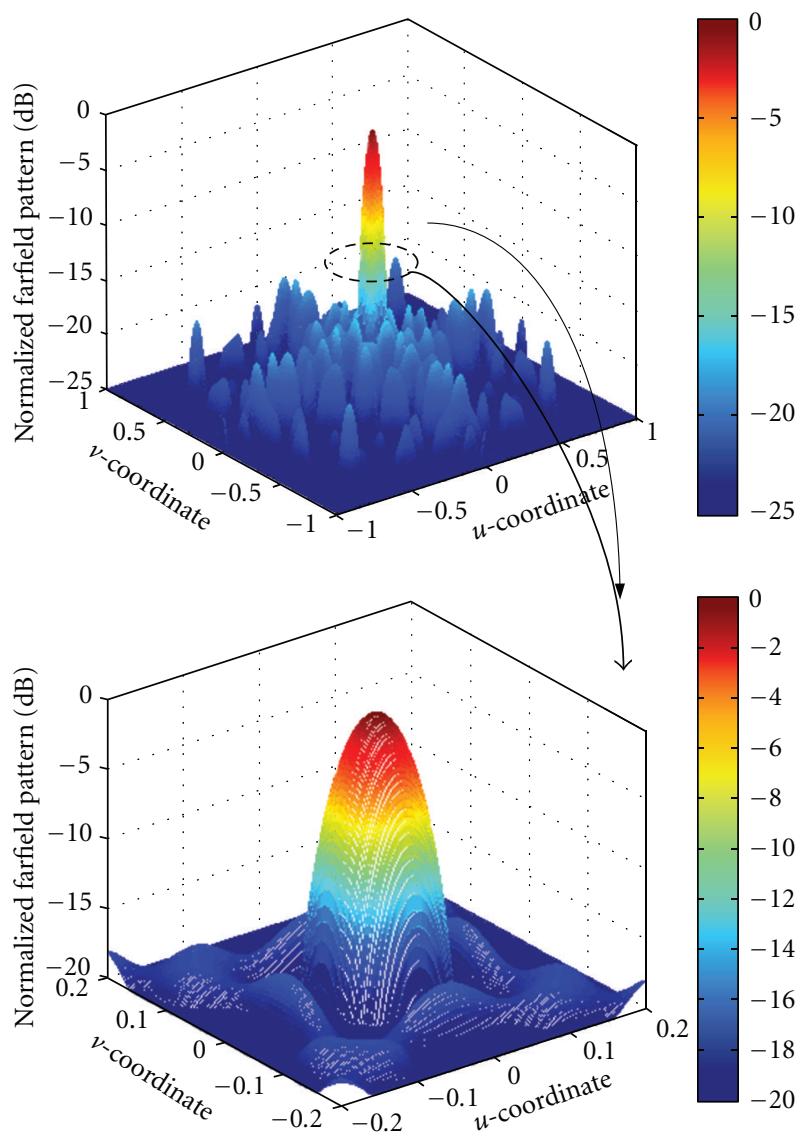

FIGURE 5: Radiation pattern of the optimized stage 3 Sierpinski carpet array.

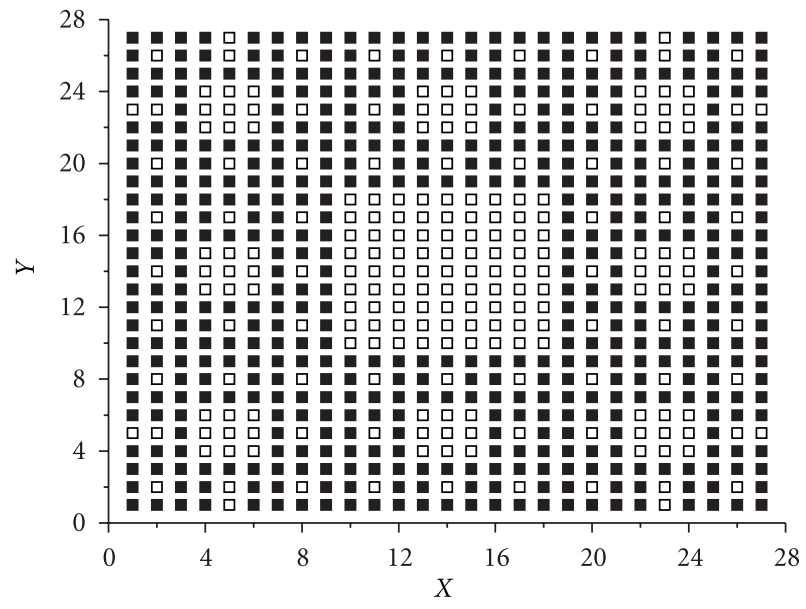

FIGURE 6: Optimized array structure for the first eaxmple.
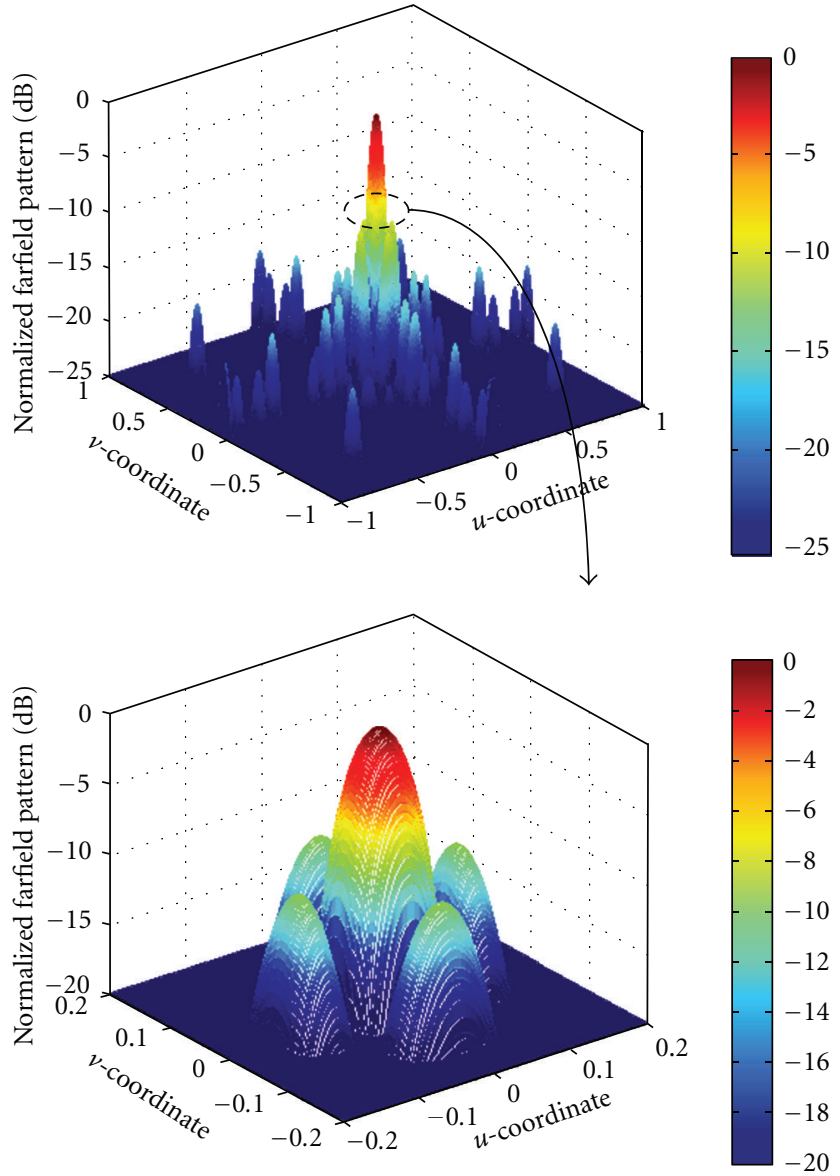

FIGURE 7: Radiation pattern of the original stage 3 Sierpinski carpet array.

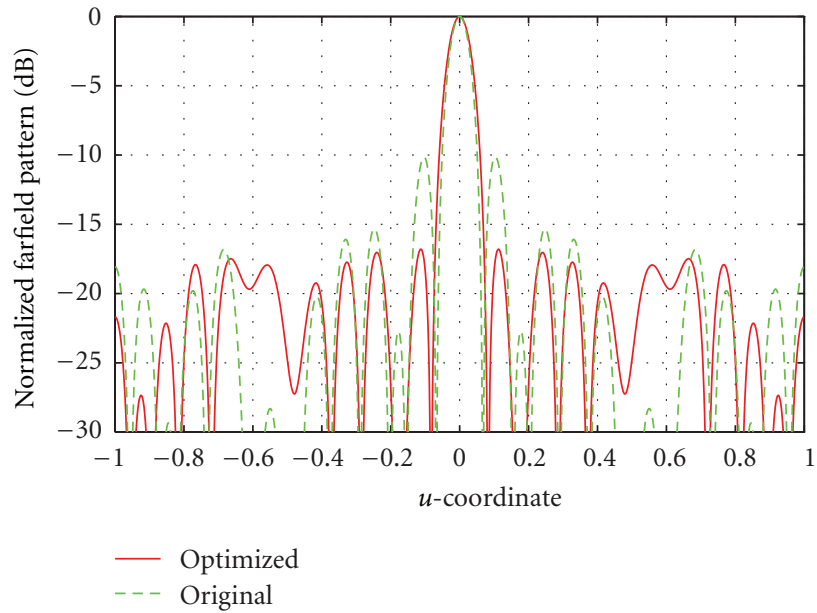

FIgURE 8: Principal $u$-cut radiation pattern for the first example. 
The sidelobes are suppressed in all planes. Furthermore, the maximum DRR was set to 5, so as to guarantee easy realization of the feeding network.

Figure 5 shows the resulting patterns. The optimized array has $-16.8 \mathrm{~dB}$ peak SLL in all planes. For verification purposes, the array structure obtained is shown in Figure 6, where the black squares represent the elements that are kept in the array, and the white squares are those eliminated from the array. For comparison, the radiation pattern of the original stage 3 Sierpinski carpet array is shown in Figure 7. The principal $u$-cut of the far-field pattern of those two arrays are illustrated in Figure 8. From the above results, it can be seen that the peak sidelobe level of the optimized array is $6.65 \mathrm{~dB}$ lower than that of the Sierpinski carpet array. What's more, although the excitation of the optimized stage 3 Sierpinski carpet array provides for a $29.8 \%$ reduction in number of elements compared with the fully filled Euclidian array, the array configuration provided in Figure 6 provides fewer elements than the original Sierpinski carpet array. Besides, the total computational time overhead to realize the result of this example is less than 30 seconds. To demonstrate the efficiency of the proposed method, the genetic algorithm is also utilized to synthesize this array, and the FFT method is incorporated into the optimization process to speed up the calculation of the fitness function for fair comparison. The iteration number is set to 3000 and the time required is more than 900 seconds. However, due to the high dimensional of the optimization problem, the genetic algorithm can hardly achieve an ideal result.

In the second example, to illustrate the flexibility of the approach, a large planar array for a stage 4 Sierpinski carpetbased array is considered, that is, the full array is a $81 \times 81$ element planar array. The IFT method to obtain the reconfigurable element distributions for this example operates with $2048 \times 2048$-point forward and inverse FFTs. The obtained patterns are presented in Figure 9, and the corresponding configuration of the array is plotted in Figure 10. The radiation pattern of the original stage 4 Sierpinski carpet array is depicted in Figure 11, while the principal $u$-cut of the farfield pattern is shown in Figure 12. The peak sidelobe level obtained by the IFT method is $-16.52 \mathrm{~dB}$, which is $-6.32 \mathrm{~dB}$ lower than the original stage 4 Sierpinski carpet thinned array. Moreover, the aperture is filled by a factor of $40.9 \%$ compared with $62.4 \%$ of the stage 4 Sierpinski carpet thinned array. Those results again demonstrate the effectiveness of the presented technique for thinned array designs. Moreover, the time overhead for IFT technique in Figure 9 is about 230 seconds. However, the genetic algorithm can hardly be used in this case due to the more complicated optimization problem. Therefore, the efficiency of the IFT technique can be testified.

\section{Conclusion}

This paper investigated the synthesis problem of designing large fractal-based weighted thinned planar arrays by using the iterative Fourier technique (IFT) method, which is used to derive element excitations from the prescribed array

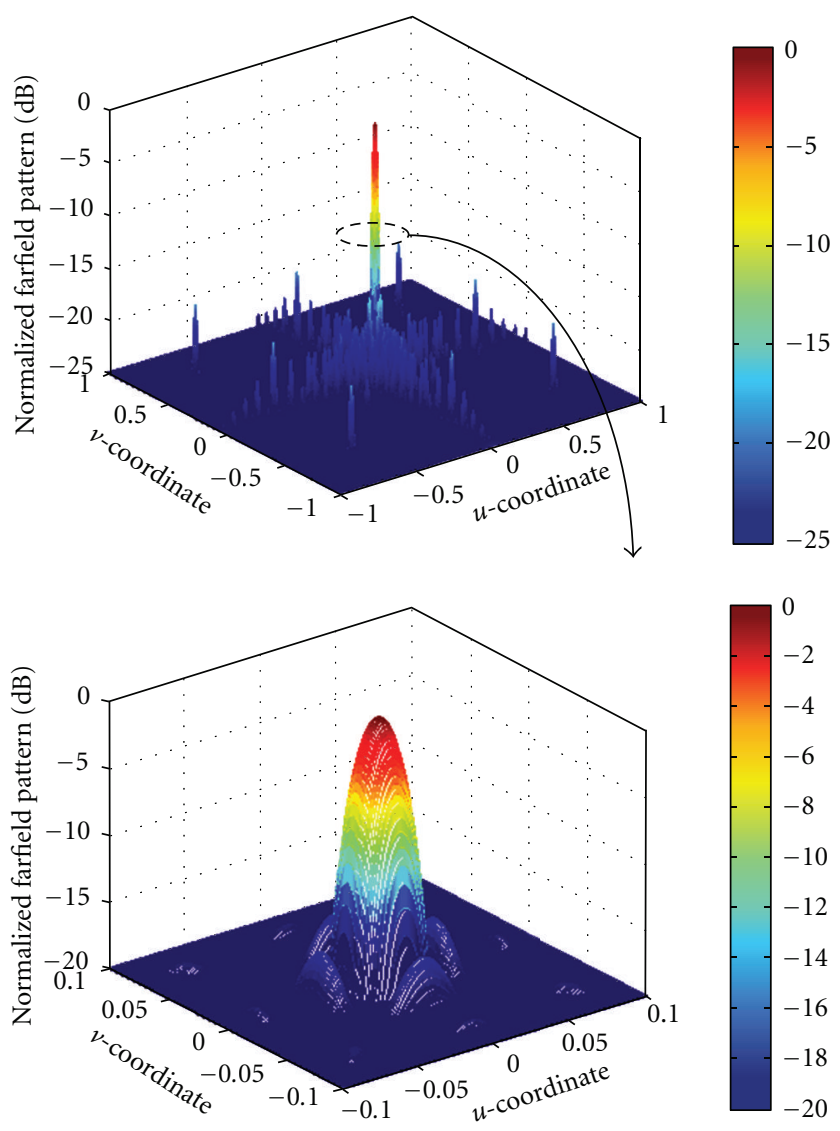

FIGURE 9: Radiation pattern of the optimized stage 4 Sierpinski carpet array.

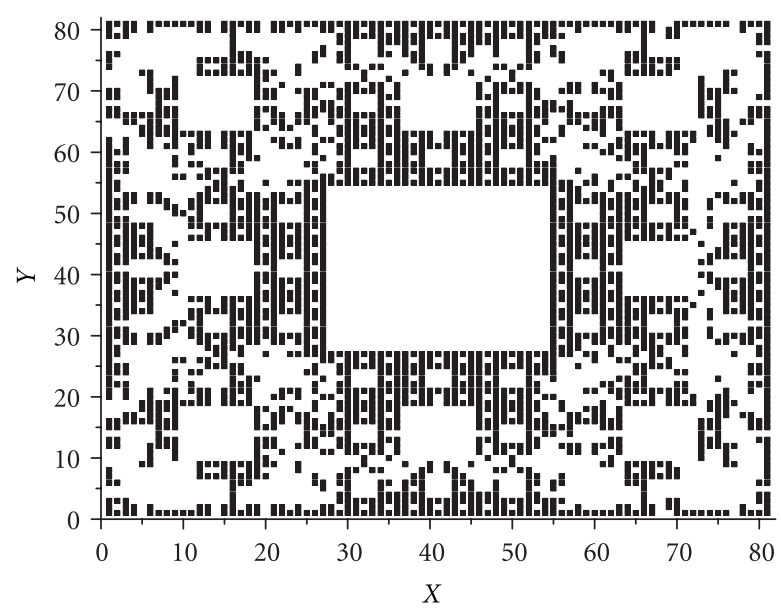

FIGURE 10: Optimized array structure for the second example.

factor. One type of fractal planar antenna array named Sierpinski carpet fractal array is investigated and modifications are made on the original Sierpinski carpet patterned array by the IFT method to obtain lower sidelobe level. Moreover, the DRR of the excitation amplitude is also controlled to allow a better control of the mutual coupling and a reduction 

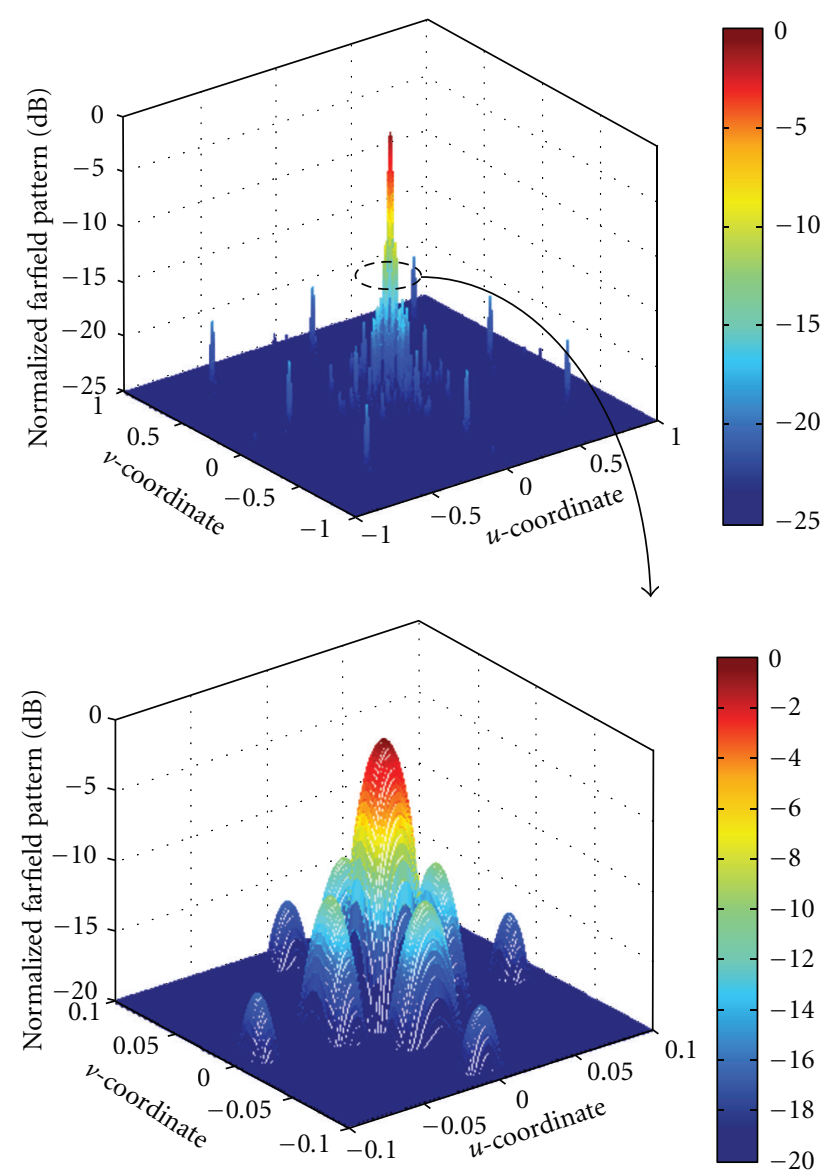

FIGURE 11: Radiation pattern of the original stage 4 Sierpinski carpet array.

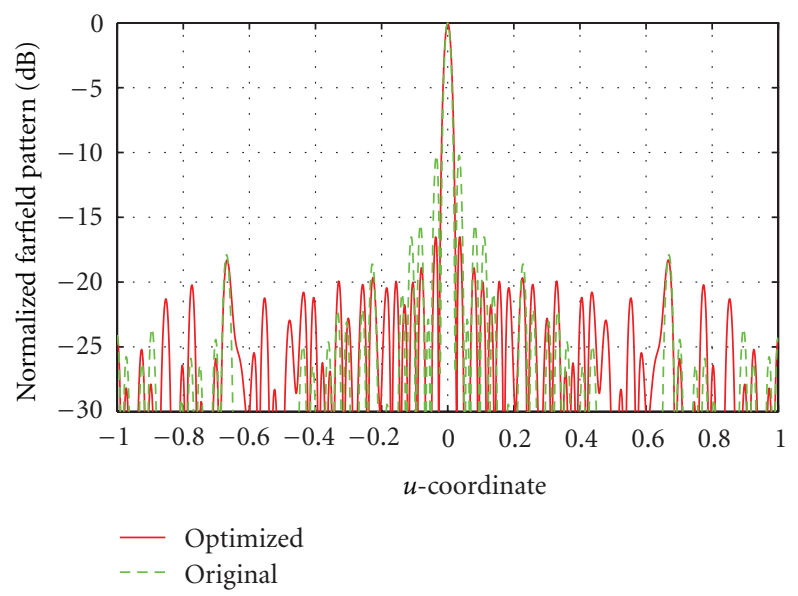

Figure 12: Principal $u$-cut radiation pattern for the second example.

of the cost of the feeding network. Numerical results reveal the effectiveness and superiority of the method.

\section{Acknowledgments}

The authors would like to thank the financial support from the Natural Science Foundation of China (no. 61101069), and the Fundamental Research Funds for the Central Universities (no. K50510010016).

\section{References}

[1] B. B. Mandelbrot, The Fractal Geometry of Nature, W. H. Freeman, New York, NY, USA, 1983.

[2] D. H. Werner and P. L. Werner, "On the synthesis of fractal radiation patterns," Radio Science, vol. 30, no. 1, pp. 29-45, 1995.

[3] L. Lizzi and G. Oliveri, "Hybrid design of a fractal-shaped GSM/UMTS antenna," Journal of Electromagnetic Waves and Applications, vol. 24, no. 5-6, pp. 707-719, 2010.

[4] A. Azari, "A new super wideband fractal microstrip antenna," IEEE Transactions on Antennas and Propagation, vol. 59, no. 5, pp. 1724-1727, 2011.

[5] R. Kumar, P. Malathi, and K. Sawant, "On the design of wheelshaped fractal antenna," Microwave and Optical Technology Letters, vol. 53, no. 1, pp. 155-158, 2011.

[6] R. Guo, X. Chen, and K. Huang, "A novel wideband microstrip fractal antenna based on the fractal binary tree," Electromagnetics, vol. 29, no. 4, pp. 283-290, 2009.

[7] J. J. Casanova, J. A. Taylor, and J. Lin, "Design of a 3-D fractal heatsink antenna," IEEE Antennas and Wireless Propagation Letters, vol. 9, pp. 1061-1064, 2010.

[8] Y. Kim and D. L. Jaggard, "The fractal random array," Proceedings of the IEEE, vol. 74, no. 9, pp. 1278-1280, 1986.

[9] C. Puente-Baliarda and R. Pous, "Fractal design of multiband and low side-lobe arrays," IEEE Transactions on Antennas and Propagation, vol. 44, no. 5, pp. 730-739, 1996.

[10] D. H. Werner, W. Kuhirun, and P. L. Werner, "The PeanoGosper fractal array," IEEE Transactions on Antennas and Propagation, vol. 51, no. 8, pp. 2063-2072, 2003.

[11] T. G. Spence, D. H. Werner, and J. N. Carvajal, "Modular broadband phased-arrays based on a nonuniform distribution of elements along the peano-gosper space-filling curve," IEEE Transactions on Antennas and Propagation, vol. 58, no. 2, pp. 600-604, 2010.

[12] J. Anguera, G. Montesinos, C. Puente, C. Borja, and J. Soler, "An undersampled high-directivity microstrip patch array with a reduced number of radiating elements inspired on the sierpinski fractal," Microwave and Optical Technology Letters, vol. 37, no. 2, pp. 100-103, 2003.

[13] F. A. Ghaffar, M. U. Khalid, K. N. Salama, and A. Shamim, "24$\mathrm{GHz}$ LTCC fractal antenna array SoP with integrated Fresnel lens," IEEE Antennas and Wireless Propagation Letters, vol. 10, pp. 705-708, 2011.

[14] D. H. Werner, R. L. Haupt, and P. L. Werner, "Fractal antenna engineering: the theory and design of fractal antenna arrays," IEEE Antennas and Propagation Magazine, vol. 41, no. 5, pp. 37-59, 1999.

[15] A. Karmakar, R. Ghatak, S. K. Mandal, R. K. Mishra, and D. R. Poddar, "A modified Sierpinski pattern thinned planar array of rectangular microstrip antenna with reduced SLL," in Proceedings of the International Workshop on Antenna Technology: Small Antennas, Novel Structures and Innovative Metamaterials (iWAT'11), pp. 384-387, Hong Kong, China, March 2011. 
[16] D. H. Werner and S. Ganguly, "An overview of fractal antenna engineering research," IEEE Antennas and Propagation Magazine, vol. 45, no. 1, pp. 38-57, 2003.

[17] D. H. Werner, K. C. Anushko, and P. L. Werner, "The generation of sum and difference patterns using fractal subarrays," Microwave and Optical Technology Letters, vol. 22, no. 1, pp. 54-57, 1999.

[18] K. K. Yan and Y. Lu, "Sidelobe reduction in array-pattern synthesis using genetic algorithm," IEEE Transactions on Antennas and Propagation, vol. 45, no. 7, pp. 1117-1122, 1997.

[19] S. K. Goudos, K. Siakavara, T. Samaras, E. E. Vafiadis, and J. N. Sahalos, "Sparse linear array synthesis with multiple constraints using differential evolution with strategy adaptation," IEEE Antennas and Wireless Propagation Letters, vol. 10, pp. 670-673, 2011.

[20] Y. J. Zhang, S. X. Gong, and Y. X. Xu, "Radiation pattern synthesis for arrays of conformal antennas mounted on an irregular curved surface using modified genetic algorithms," Journal of Electromagnetic Waves and Applications, vol. 23, no. 10, pp. 1255-1264, 2009.

[21] J. L. Guo and J. Y. Li, "Pattern synthesis of conformal array antenna in the presence of platform using differential evolution algorithm," IEEE Transactions on Antennas and Propagation, vol. 57, no. 9, pp. 2615-2621, 2009.

[22] S. W. Yang, Y. B. Gan, and A. Y. Qing, "Antenna-array pattern nulling using a differential evolution algorithm," International Journal of RF and Microwave Computer-Aided Engineering, vol. 14, no. 1, pp. 57-63, 2004.

[23] T. H. Ismail and Z. M. Hamici, "Array pattern synthesis using digital phase control by quantized particle swarm optimization," IEEE Transactions on Antennas and Propagation, vol. 58, no. 6, pp. 2142-2145, 2010.

[24] D. W. Boeringer and D. H. Werner, "Particle swarm optimization versus genetic algorithms for phased array synthesis," IEEE Transactions on Antennas and Propagation, vol. 52, no. 3, pp. 771-779, 2004.

[25] W. P. M. N. Keizer, "Linear array thinning using iterative FFT techniques," IEEE Transactions on Antennas and Propagation, vol. 56, no. 8, pp. 2757-2760, 2008.

[26] W. P. M. N. Keizer, "APAS: an advanced phased-array simulator," IEEE Antennas and Propagation Magazine, vol. 52, no. 2, pp. 40-56, 2010. 

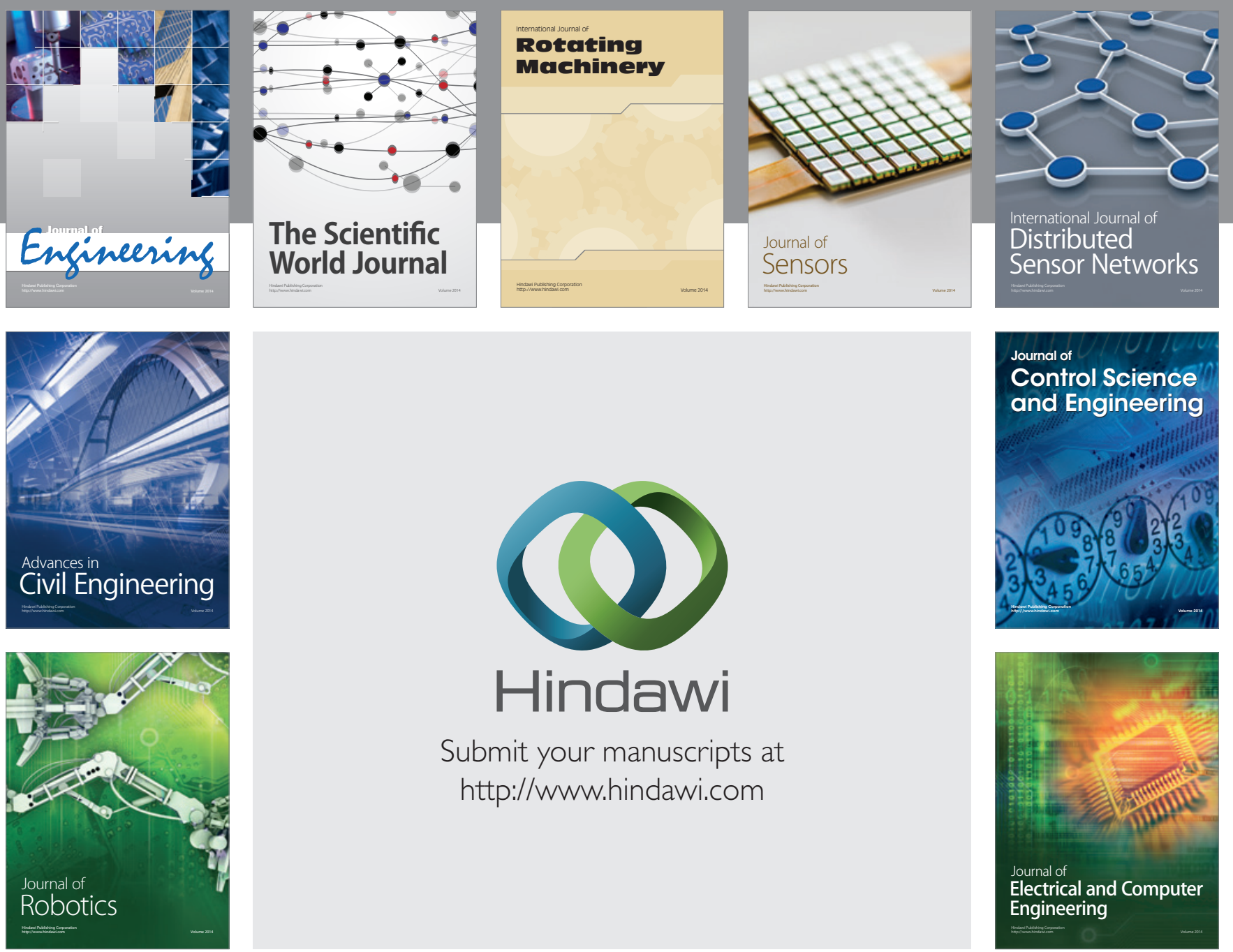

Submit your manuscripts at

http://www.hindawi.com
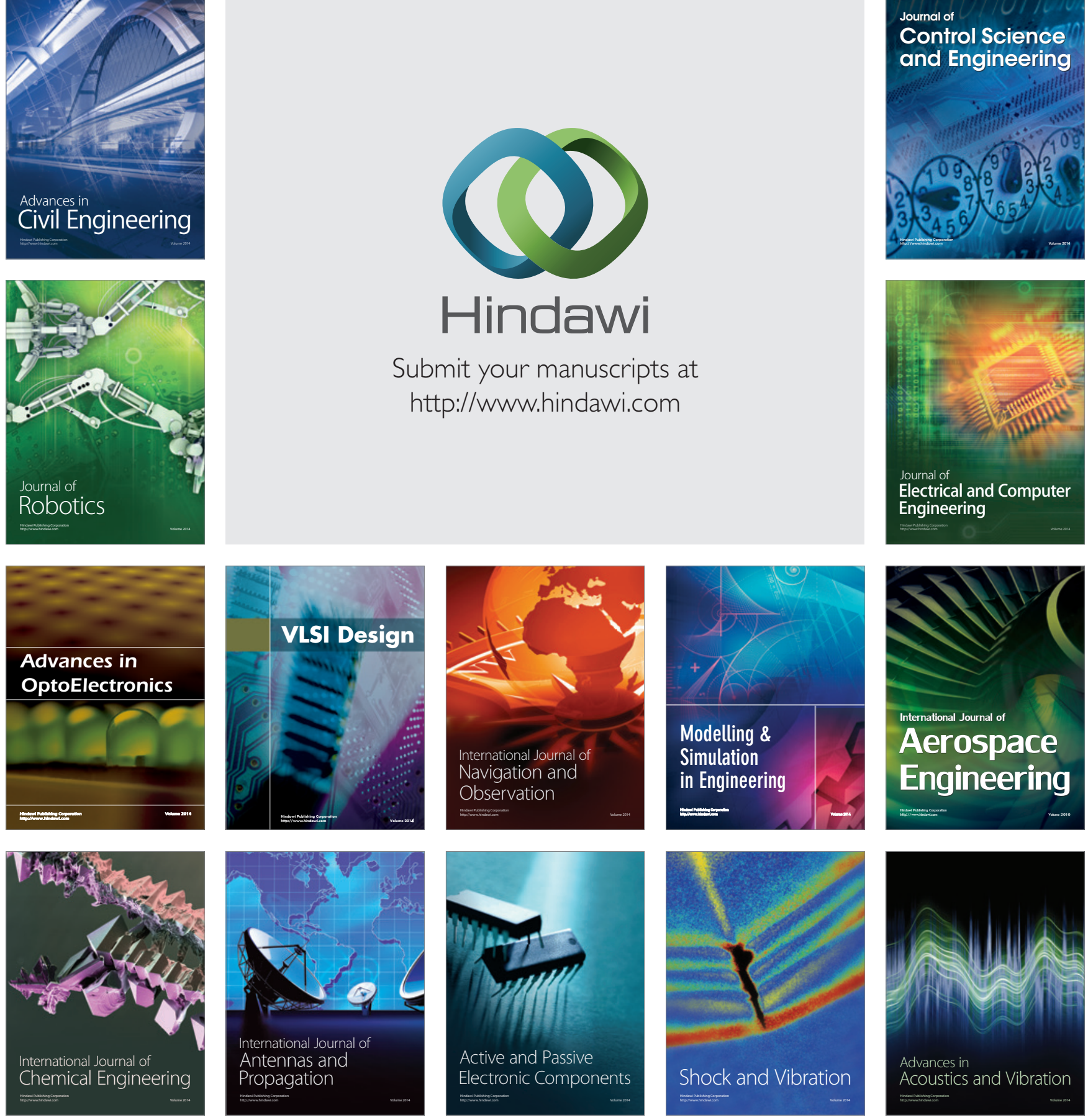